\title{
Selected news items and updates for the practicing clinician
}

\author{
Zachary Mulkey MD
}

- The FDA is planning to extend its regulatory authority to electronic cigarettes and the nicotine solutions used for vaping. There is a period of comment now and the public is encouraged to read the rule and submit comments here.

- An updated systematic review is reporting that weaning via non-invasive ventilation continues to offer benefits over continued invasive ventilation, including mortality.

- Could protocol-based care for septic shock be no better than "usual care" because our "usual care" is so much better than it was just 10 years ago when the Surviving Sepsis campaign was getting off the ground?

- Inhaled insulin is getting another opportunity by the FDA.

- According to the $\mathbf{W H O}$, Middle East coronavirus is a serious concern (50\% of patients have died) but not a global health emergency (only 50 cases identified since late 2012). 